\title{
Jogos Lúdicos sob a ótica do Pensamento Computacional: Experiências do Projeto Logicamente
}

\author{
Graziela Ferreira Guarda ${ }^{1}$, Ione Ferrarini Goulart ${ }^{2}$ \\ ${ }^{1}$ Departamento de Computação - Universidade Católica de Brasília (UCB) \\ Campus I - QS 07 - Lote 01 - EPCT - Águas Claras - Brasília - DF CEP: 71966-700. \\ ${ }^{2}$ Área de Informação e Comunicação - Instituto Federal de Brasília (IFB) \\ Campus Brasília - SGAN 610 Módulo D, E, F e G - CEP: 70830-450. \\ grazielafguarda@gmail.com, ionefgegmail.com
}

\begin{abstract}
This article describes a methodology for the use of playful tools supported by Declined Computing as a teaching-learning process for Basic Education from the activities of the research project. Logically, the purpose of this study is to study and seek alternatives for the construction of a teachinglearning process, which seeks to contribute to the insertion of new methods and improvement of school performance in the context of Exact Sciences from the perspective of Computational Thinking. Considering the Brazilian school scenario, learning objects were constructed with accessible materials to work the insertion of skills such as assembly of logical sequences, programming logic, cryptography, among others. In the end, it was possible to identify significant improvements in the school performance of students.
\end{abstract}

Resumo. Esse artigo descreve uma metodologia para uso de ferramentas lúdicas amparada pela Computação Desplugada como processo de ensinoaprendizagem para a Educação Básica oriundo das atividades do projeto de pesquisa Logicamente cujo propósito é estudar e buscar alternativas para a construção de um processo de ensino-aprendizagem gamificado que visa contribuir para a inserção de novos métodos e melhoria do rendimento escolar no contexto das Ciências Exatas sob a ótica do Pensamento Computacional. Considerando o cenário escolar brasileiro, foram construídos objetos de aprendizagem com materiais acessíveis para trabalhar a inserção de habilidades como montagem de sequências lógicas, lógica de programação, criptografia, entre outros. Ao final, foi possivel identificar melhoras significativas no rendimento escolar dos estudantes atendidos.

\section{Introdução}

Nos últimos anos, são notórios os esforços e o interesse pela pesquisa e desenvolvimento de recursos tecnológicos voltados a apoiar os processos de ensinoaprendizagem. Em especial, se destacam as ferramentas computacionais que oferecem ambientes lúdicos e interativos, capazes de atrair a atenção de crianças e adolescentes, aumentando o interesse e favorecendo a aprendizagem de conteúdos escolares.

$\mathrm{Na}$ busca pela inovação, criatividade e motivação nas práticas pedagógicas, jogos educativos - que podem ser digitais ou desplugados - têm sido considerados um importante recurso tecnológico de apoio à educação, uma vez que estes aumentam o engajamento e a motivação do aprendiz. Além disso, a diversão inerente ao ambiente 
VII Congresso Brasileiro de Informática na Educação (CBIE 2018)

Anais do XXIX Simpósio Brasileiro de Informática na Educação (SBIE 2018)

lúdico de um jogo amplia as possibilidades de interesse e absorção de conteúdos educacionais.

Diversos trabalhos propõem o uso de jogos no ensino de programação e aplicação do pensamento computacional (PC) não só para estudantes da área de tecnologia, mas também no âmbito da Educação Básica e tal prática não tem como objetivo o preparo de uma habilidade profissional, mas sim, o despertar do desenvolvimento de competências que visam a melhora de outras áreas do conhecimento. (Andrade et al., 2013), (Campos et al., 2014), (Pinho et al., 2016), (Silva et al., 2016) destacam que aprender a programar é uma tarefa difícil e complexa para iniciantes e que a abordagem com uso de jogos é um caminho facilitador desse aprendizado.

O ensino de algoritmos e linguagem de programação busca nas Ciências Exatas um pilar de sustentação, pois disciplinas nessa área despertam o raciocínio lógico para resolução de problemas (Santos et al., 2006). Por essas razões, têm sido testadas técnicas e formas de inclusão do ensino de programação o mais cedo possível, seja na Educação Fundamental ou Ensino Médio, podendo abranger inclusive a Educação Infantil.

Diante do exposto, o problema abordado neste artigo consiste em investigar e avaliar uma nova forma de abordagem para inserção da lógica de programação no Ensino Fundamental como forma de despertar e/ou fortalecer o raciocínio lógicomatemático dos estudantes. Neste sentido, surge a proposta da criação de jogos lúdicos como potencial ferramenta de apoio à aprendizagem, aliado a uma estratégia de integração entre universidade e escola.

Deste modo, o presente trabalho apresenta uma abordagem para a construção de um processo de ensino-aprendizagem gamificado, visando a inclusão do ensino de programação e algoritmos no Ensino Fundamental através da realização de atividades lúdicas gamificadas usando recursos informacionais e ferramentas educacionais diferenciadas e inovadoras de forma ágil e divertida. Como objetivos específicos, se destacam: a) despertar nos estudantes o interesse pela programação; b) trabalhar o raciocínio lógico-matemático dos estudantes - pilar das Ciências Exatas; c) realizar atividades lúdicas, no contexto da Computação Desplugada sob a ótica do $\mathrm{PC}$; d) identificar os aspectos positivos e negativos das experiências práticas, bem como, quais competências e habilidades do PC estão sendo trabalhadas; e) aproximar universidade e escola.

Essa abordagem foi elaborada pela Universidade Católica de Brasília (UCB), nos anos de 2016 a 2017, no âmbito do ensino e pesquisa junto a uma escola particular da cidade de Brasília, DF cujo público-alvo foi - no primeiro ano de implementação estudantes dos $5^{\circ}$ e $6^{\circ}$ anos do Ensino Fundamental.

O presente trabalho está dividido da seguinte maneira: a seguir, na Seção 2 será apresentado parte do embasamento teórico utilizado pelo projeto Logicamente. $\mathrm{Na}$ Seção 3 é apresentada a metodologia utilizada e os Objetos de Aprendizagem (OAs) criados. Os resultados parciais são descritos na Seção 4. Por fim, os objetivos e metas desta experiência serão destacados na Seção 5 , de forma a concluir o propósito do 
VII Congresso Brasileiro de Informática na Educação (CBIE 2018)

Anais do XXIX Simpósio Brasileiro de Informática na Educação (SBIE 2018)

presente trabalho diante dos resultados já obtidos, bem como, relatar a perspectiva de resultados futuros e melhorias que poderão ser integradas ao projeto posteriormente.

\section{Trabalhos Relacionados}

No contexto do uso de jogos como apoio ao processo de ensino-aprendizagem no contexto da computação, [Andrade et al. 2013] propôs um conjunto de atividades lúdicas para estudantes do Ensino Fundamental, onde foram abordados nove conceitos considerados essenciais para o desenvolvimento do PC nas escolas. As atividades foram os jogos Cara a Cara, Caça ao Tesouro e Organização de Festa. Depois de executados os trabalhos, foram discutidos junto aos estudantes, os meios utilizados na realização dos jogos e foram analisadas as técnicas do PC para cumprir as tarefas de modo eficiente.

[Campos et al. 2014] apresentou de uma proposta metodológica para o ensino dos números binários para desenvolver o $\mathrm{PC}$ com crianças do $4^{\circ}$ ano. As atividades foram fundamentadas na Computação Desplugada, onde com o uso de cartas, realizaram tarefas como conversão de números binários para decimal, de letras e palavras para números binários e vice-versa. Os resultados foram expostos de forma quantitativa com vários critérios de avaliação adotados pelos pesquisadores, onde foram avaliados positivamente quanto ao incentivo do $\mathrm{PC}$.

[Pinho et al. 2016] visando aumentar o interesse dos estudantes por algoritmos, descreve a proposta e aplicação de uma atividade que introduz os conceitos elementares de algoritmos por meio de jogos de tabuleiro e de um jogo intitulado Salve a Princesa, aplicado a estudantes do $5^{\circ}$ ano de escolas públicas. Os resultados foram mensurados por aplicação de testes antes e depois das atividades e foram considerados satisfatórios.

[Pinho et al. 2016] também mostram os resultados da atividade Caça ao Tesouro, onde aborda as premissas de busca de dados, utilizadas nos conceitos de Ciência da Computação. Para executar essa atividade, os autores usaram os conceitos da Computação Desplugada, que permitiu depois, desenvolver um jogo educativo digital. Foram avaliados os jogos nas formas clássica e digital, sendo comparados os resultados nas duas formas.

[Silva et al. 2016] abordou o uso da robótica para o ensino de lógica de programação e estimulação o PC. A metodologia apresentada pelos autores incluiu a utilização da tecnologia Lego Mindstorms e do arduíno como OAs para auxiliar nas atividades. Primeiramente, foram apresentados os conceitos sobre Robótica e Programação, para em seguida, aplicarem atividades com o kit Lego. Para concluir o projeto, foi realizado um projeto com o arduíno. Observou-se que o uso da robótica educativa despertou o interesse científico nos estudantes e os incentivou à resolução de problemas.

Os estudos realizados foram de suma importância para a construção da metodologia e definição dos OAs a serem utilizados pelo projeto Logicamente. Os estudos visaram em sua maioria o desenvolvimento do raciocínio lógico, por meio das técnicas propostas por (Wing 2006), criadora do PC. Na maioria dos trabalhos analisados, foram usadas pesquisas comparativas com a intenção de mensurar a influência do PC na aprendizagem de outras disciplinas, sempre chegando à conclusão que o PC pode realmente aprimorar o raciocínio lógico dos participantes. 
VII Congresso Brasileiro de Informática na Educação (CBIE 2018)

Anais do XXIX Simpósio Brasileiro de Informática na Educação (SBIE 2018)

\section{Projeto Logicamente: Proposta, Jogos Lúdicos e Metodologia}

O projeto Logicamente surgiu oriundo da pretensão de contribuir para o despertar do interesse de estudantes da Educação Básica pelas carreiras de Ciências \& Tecnologia. As estatísticas mostram que os percentuais de matrículas nos cursos das áreas de Ciências Exatas são baixos, especificamente no caso dos cursos de Computação, esse número abaixa ainda mais para em torno de $2 \%$ de acordo com os dados do censo do Ensino Superior Brasileiro (INEP, 2016).

Neste contexto, se estabeleceu uma parceria entre a UCB e uma escola particular do DF para implementar ações de âmbito computacional atendendo estudantes do Ensino Fundamental com uma proposta em formato de reforço pedagógico. Deste modo, ficou definido em consenso entre a equipe do projeto Logicamente e a equipe pedagógica da escola que os anos atendidos seriam o $5^{\circ}$ e $6^{\circ}$ cujas idades dos estudantes variam entre 9 e 11 anos. Ao final, foram atendidos 35 estudantes do $5^{\circ}$ ano e 18 do $6^{\circ}$ ano e os mesmos foram divididos em três turmas, totalizando 53 participantes. A escolha pelos $5^{\circ}$ e $6^{\circ}$ anos foi determinada pelas idades consideradas mais adequadas pela equipe pedagógica da escola a receber os reforços pedagógicos.

Para fins de levantamento dos conteúdos a serem abordados nos jogos lúdicos criados pela equipe do projeto, se realizou a leitura dos planos de aula anual das séries atendidas, onde foram identificados quais assuntos os estudantes iriam estudar durante o ano letivo. Dentre eles se destacam: Operações básicas (somas, subtrações, multiplicações e divisões), potências, raízes quadradas, noções de geometria, frações, polígonos e unidades de área, de volume e de massa. Após esse levantamento a equipe do projeto construiu três lúdicos conforme descritos abaixo:

\subsection{CriptoLAB}

Tem por objetivo contribuir para a construção de um processo de ensino-aprendizagem gamificado, no qual o desenvolvimento do raciocínio lógico e computacional são estimulados através de práticas que abordam a criptografia e uso de sequencias lógicas de forma estruturada amparada pela computação desplugada.

O jogo foi estruturado em cinco partes, sendo que, as quatro primeiras abrangem as mesmas orientações: sortear e responder uma questão, decodificar uma mensagem, procurar uma ficha e montar partes de um esquema lógico - programa desplugado baseado na linguagem do aplicativo Scratch do MIT. A conclusão das primeiras 4 partes possibilita a travessia de um labirinto impresso numa folha de papel A4, que se refere a quinta parte do jogo.

A primeira etapa do jogo se refere ao sorteio de uma questão que deveria ser respondida pelas equipes. As perguntas foram de múltipla escolha e tinham como assuntos o raciocínio lógico-matemático alinhado com os conhecimentos acadêmicos dos estudantes de $5^{\circ}$ e $6^{\circ}$ anos. As questões foram retiradas e adaptadas de sítios de concursos públicos e desafios. Assim que finalizada as respostas, as mesmas eram corrigidas e, estando corretas, a próxima fase era desbloqueada.

A segunda etapa do jogo, consistiu em decodificar uma mensagem, cujo conteúdo revelava qual funcionário da escola estaria com a ficha responsável por liberar a etapa seguinte (trabalho com criptografia), já na terceira etapa, o estudante "corredor" trocava a ficha pelo envelope com os trechos de código que tornaram possível a 
VII Congresso Brasileiro de Informática na Educação (CBIE 2018)

Anais do XXIX Simpósio Brasileiro de Informática na Educação (SBIE 2018)

passagem pelo labirinto. Posteriormente, a quarta etapa se refere à montagem do código de modo que, o acesso à próxima parte do jogo só será permitido se a construção do mesmo estiver correta. Esse conjunto de etapas se repetiu quatro vezes, mudando apenas o conteúdo. Ao final, os grupos receberam uma folha com o labirinto e com a tabela que deveria ser escrita a solução do problema (Fig. 1), que consistia em expor quantas vezes e quais seriam as teclas que devem ser acionadas, simulando como se a atividade fosse no computador (computação desplugada).

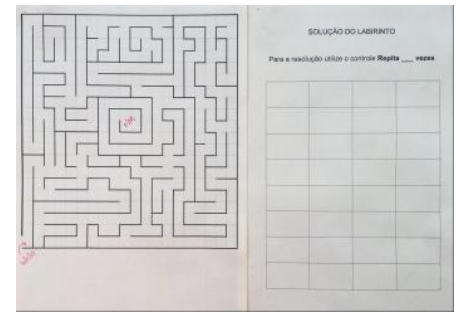

Figura 1. Labirinto e Tabela de Solução

O CriptoLab foi jogado por um total de quarto equipes, cada equipe continha entre 4 e 5 integrantes. Cada grupo se reuniu em um canto da sala e, antes do jogo começar, cada um deles escolheu um representante para sortear as questões e outro para exercer a função de "corredor", o corredor tinha a responsabilidade de correr atrás dos funcionários da escola que estavam com quatro fichas para recolhê-las, posteriormente, as fichas seriam trocadas pelos envelopes que continha trechos de código baseado no aplicativo Scratch, que seriam utilizadas na etapa seguinte conforme exposto na Fig. 2 a esquerda acima os envelopes, a esquerda abaixo parte de trechos de código e a direita, o esquema lógico montado por uma das equipes. Foi estabelecido como regra geral que cada fase não poderia ultrapassar 20 minutos e esse tempo foi cronometrado.

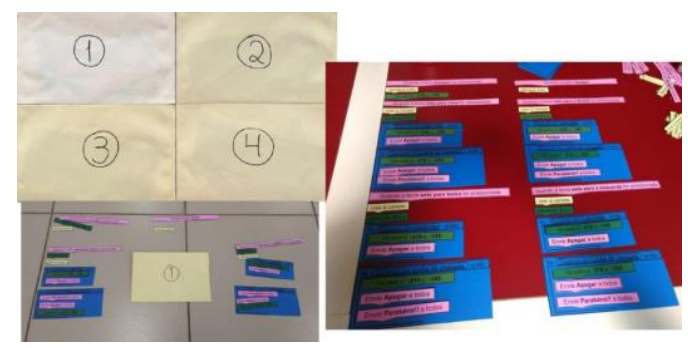

Figura 2. Etapas do Jogo

Materiais utilizados: Envelopes de papel, cartolina, papéis A4 coloridos para montagens das sequencias lógicas, papel A4 branco.

\subsection{LightBot Logicamente:}

Tem por objetivo contribuir para a construção de um processo de ensino-aprendizagem gamificado, inspirado no jogo digital educativo LightBot cujo enfoque foi induzir o conhecimento de raciocínio lógico ensinando comandos básicos de programação através do uso de comandos que fazem referência direta aos algoritmos explorando as estruturas sequenciais, de seleção, de repetição e funções/procedimentos sem a necessidade de compreensão de escrita de pseudocódigos. A versão lúdica desplugada contou com regras diferenciadas contendo um adicional de jogadas com grau de complexidade mais amplo para atender as expectativas dos estudantes. 
VII Congresso Brasileiro de Informática na Educação (CBIE 2018)

Anais do XXIX Simpósio Brasileiro de Informática na Educação (SBIE 2018)

O jogo contou com um robô cuja função era realizar um percurso dentro de um labirinto com determinado número de jogadas. O jogador visualizava um bloco de ações principal e outros dois de ações secundárias - PROC1 e PROC2. Era permitido que o robô executasse comandos como: andar para frente, virar à direita, virar à esquerda, pular, acender a luz e acionar procedimentos repetitivos (PROC1 e PROC2). Essas orientações ou conjunto de ações que o robô poderia executar deveriam ser informados no bloco Main utilizando os comandos (Fig. 3) para montar a sequência lógica a ser executada.

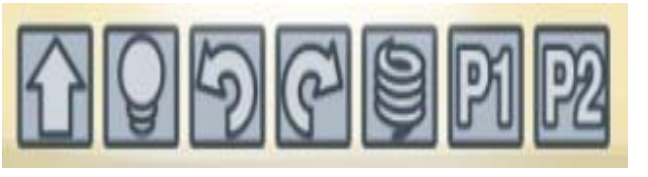

Figura 3. Comandos LightBot Versão original

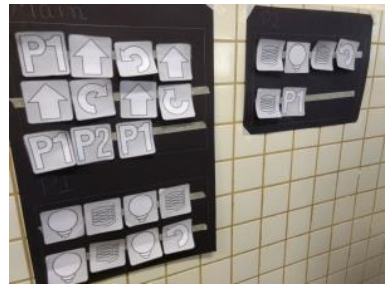

Figura 4. Comandos LightBot Logicamente

A versão lúdica foi estruturada com utilização de dois quadros para que os estudantes pudessem recolher e selecionar os blocos para posteriormente montar as sequências lógicas determinadas (Fig 4). Como regra, ficou estabelecido que os estudantes só poderiam utilizar 12 comandos no bloco Principal, 8 no bloco P1 e 8 no bloco P2, dificultando a solução. Além disso, para o jogo ficar mais interessante e fortalecer os conteúdos da área da matemática, ficou decidido que, substituindo a ação de acender a luz, deveria ser respondido uma pergunta da Olimpíada Brasileira de Matemática referente ao Ensino Fundamental (OBM).

O jogo contou com a divisão da turma em grupos de quatro integrantes. Todos tiveram 65 minutos para realização da atividade, de modo que neste tempo, deveriam achar uma solução para o tabuleiro, e em seguida, responder às questões da OBM. O grupo que terminasse primeiro o tabuleiro e respondesse as questões (sem verificação do gabarito/correção) no menor tempo ganharia o jogo. Para fins de critério de desempate, venceria o grupo que respondesse o maior número de questões corretamente.

Materiais utilizados: Isopor para montagem do tabuleiro, boneco feito de biscuit (robô), cartolinas pretas para montar o quadro com os comandos, papéis A4.

\subsection{Circuito 4 Desafios:}

Tem por objetivo desenvolver o raciocínio lógico e matemático alinhado com as habilidades e competências advindas do PC amparado pela ludicidade como ferramenta do desenvolvimento do aprendizado. Para este lúdico, a sala foi dividida em quatro áreas, cada uma representada por uma cor e um número com o propósito de deixar o ambiente mais organizado, manter a privacidade dos grupos, bem como, não prejudicar a concentração dos estudantes conforme ilustrado na Fig. 5.

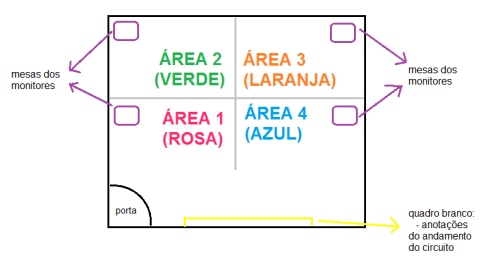


VII Congresso Brasileiro de Informática na Educação (CBIE 2018)

Anais do XXIX Simpósio Brasileiro de Informática na Educação (SBIE 2018)

\section{Figura 5. Organização do Ambiente}

Cada área dispôs de uma atividade diferente e foi acompanhada por um monitor que exerceu a função de auxiliar os grupos para fins de esclarecimento de dúvidas. Paralelamente, os monitores ficaram responsáveis, ainda, por avaliar o desempenho dos estudantes e o desenvolvimento da atividade, observando sua efetividade e a capacidade de abstração dos estudantes. Quanto a dinâmica utilizada, o tempo total destinado a execução da atividade foi 100 minutos. Para cada grupo foi definido um líder, a função do líder era receber as medalhas (que teria uma pontuação). Cada equipe tinha 20 minutos para resolver cada parte do circuito. Ao final dos 20 minutos, independente da conclusão do desafio daquela área, as equipes avançaram para a próxima área trocando de atividade no sentido horário.

Ao concluir o desafio de cada área, o líder da equipe ganhava uma medalha que valia 400 pontos. Como critério de penalização, alguns objetos foram definidos: equipes que produzissem barulho excessivo perdia 50 pontos; equipes que copiasse a solução de outra equipe perdia 100 pontos. Nos casos de empate, seria realizada uma prova surpresa. Em relação a atividade em si, os desafios do circuito foram divido em quatro áreas descritas abaixo:

Desafio do Tangram: Área 1 (rosa). Inspirado nos antigos quebra cabeças chineses é um jogo composto por sete peças geométricas, são elas: dois triângulos isósceles grandes, dois pequenos, um médio, um quadrado, um paralelogramo. Esse quebra cabeça pode formar várias figuras e formas que podem ser de animais, pessoas, objetos até outras figuras geométricas. Desenvolvendo a abstração, reconhecimento e manipulação de figuras geométricas.

Desafio do Resta 1: Área 2 (verde). O Resta 1 tradicional (ou Solitaire) é um jogo inglês formado por um tabuleiro com trinta e três casas, dispostas em forma de cruz onde 32 casas são ocupadas pelas peças do jogo, deixando a casa central vazia. $\mathrm{O}$ objetivo do jogo é retirar as peças, com um movimento semelhante ao jogo de damas, de forma a sobrar somente uma peça - casa do centro do tabuleiro.

Desafio da Expressão Numérica: Área 3 (laranja). Com o objetivo desenvolver a habilidade de solucionar um problema através da decomposição, ou seja, pegar um problema complexo e dividi-lo em partes menores, foi aplicado no circuito a uma expressão numérica.

Desafio do fazendeiro: Área 4 (Azul). Nesta área foi apresentado o seguinte problema: "Um fazendeiro quer levar o leão, o burro e o feno para o outro lado do rio. Porém, ele não pode levar o leão junto com o burro, pois este o comeria. Nem o burro com o feno.". Solucionado o problema, era entregue ao grupo um papel que os estudantes deveriam escrever um algoritmo que representasse a solução do problema.

Materiais utilizados: Tangram: Folhas de papel A4 com a sombra da figuras e peças de papel A4 do Tangram coloridas; Resta Um: Folhas de papel A4 com a marcação do tabuleiro e círculos de papel cartão para representar as peças; Expressão Numérica: Folhas de papel A4, lápis e borracha; Desafio do Fazendeiro: Uma maquete do cenário (margens do rio), cartões para escrever a solução e origamis de leão, burro e barco, além da representação feno. 
VII Congresso Brasileiro de Informática na Educação (CBIE 2018)

Anais do XXIX Simpósio Brasileiro de Informática na Educação (SBIE 2018)

\section{Experiências com o desenvolvimento do projeto e resultados parciais}

As atividades do projeto Logicamente se encontram em desenvolvimento. No primeiro ano de implementação (2017) os três OAs expostos na seção 3 foram utilizados com os estudantes do Ensino Fundamental e dessa experiência algumas percepções foram identificadas através das observações realizadas.

Em relação ao PC, a International Society for Technology in Education (ISTE) lista um conjunto de nove habilidades aplicadas no contexto da educação norteamericana que são conhecidas como núcleo do PC, que de acordo com (Barr et at., 2011) são: Abstração: capacidade de filtrar informações essenciais e descartar as informações desnecessárias em um determinado contexto; Decomposição: quebrar um problema grande em subproblemas, facilitando sua solução; Automação: utilizar a tecnologia como meio na resolução de tarefas repetitivas; Coleta de Dados: encontrar os dados necessários para resolver um problema; Representação de Dados: usar estruturas de dados, como tabelas e gráficos, para apresentar os dados; Análise de Dados: atribuir sentido a dados, encontrando padrões e conclusões; Construção de Algoritmo: sequências lógica de instruções para alcançar um objetivo; Paralelismo: identificar atividades que podem ser executadas em paralelo; Simulação: Usar ou criar simulações para experimentos. A Tabela 5 demonstra quais habilidades foram trabalhadas em cada lúdico:

Tabela 5. Conteúdos e Habilidades do PC trabalhadas nos Lúdicos

\begin{tabular}{|c|c|}
\hline OA: & Habilidades PC: \\
\hline CriptoLab & Abstração, Decomposição, Coleta de Dados, Análise de Dados e Construção de Algoritmo. \\
\hline $\begin{array}{l}\text { ot } \\
\text { mente }\end{array}$ & $\begin{array}{l}\text { Automação, Decomposição, Coleta de Dados, Análise de Dados e Construção de } \\
\text { Algoritmo. }\end{array}$ \\
\hline $\begin{array}{ll}\text { Circuito } & 4 \\
\text { Desafios } & \end{array}$ & $\begin{array}{l}\text { Tangram: Abstração; Análise de Dados; Construção de Algoritmo. } \\
\text { Resta Um: Abstração; Construção de Algoritmo; Paralelismo. } \\
\text { Expressão Numérica: Decomposição; Abstração; Paralelismo. } \\
\text { Desafio do Fazendeiro: Coleta de dados; Análise de Dados; Construção do Algoritmo. }\end{array}$ \\
\hline
\end{tabular}

De acordo com os dados expostos pela Tabela 5, os lúdicos trabalharam seis das nove habilidades do PC. Em relação aos aspectos positivos e as dificuldades apresentadas, segue síntese das percepções observadas:

CriptoLab: Aspectos Positivos: Todas as equipes conseguiram concluir as 5 fases do jogo com sucesso dentro do tempo estabelecido. A divisão do problema em partes menores facilitou o trabalho das equipes. Em relação ao aprendizado dos conteúdos, se observou que os estudantes que se dedicaram a aprender sobre o tema anteriormente a dinâmica, passaram pelas fases com relativa facilidade, inclusão do tema criptografia. Dificuldades: Em relação ao raciocínio lógico-matemático, em relação a montagem das sequencias do código fonte.

LightBot Logicamente: Aspectos Positivos: Complexidade mais aprimorada para conclusão das etapas e trabalho em equipe. Dificuldades: Montagem das sequências lógicas.

Circuito 4 Desafios: Aspectos Positivos: Trabalho em equipe, as equipes desenvolveram o raciocínio correto no tempo estipulado, acertaram os desafios. Desenvolvimento das 
VII Congresso Brasileiro de Informática na Educação (CBIE 2018)

Anais do XXIX Simpósio Brasileiro de Informática na Educação (SBIE 2018)

relações interpessoais, os estudantes se mostraram motivados durante a realização das atividades despertando positivamente a competitividade entre os grupos. Dificuldades: Cálculos matemáticos, divisão das tarefas.

Em paralelo, outro parâmetro foi analisado na compilação dos dados parciais do projeto. A escola forneceu o relatório de notas escolares (boletins) dos estudantes atendidos, de modo que, foi possível fazer um diagnóstico da evolução das notas conforme demonstrados nos Gráficos 1 e 2 abaixo - os dados foram divididos por ano atendido - resultados do $5^{\circ}$ ano e $6^{\circ}$ ano separadamente:

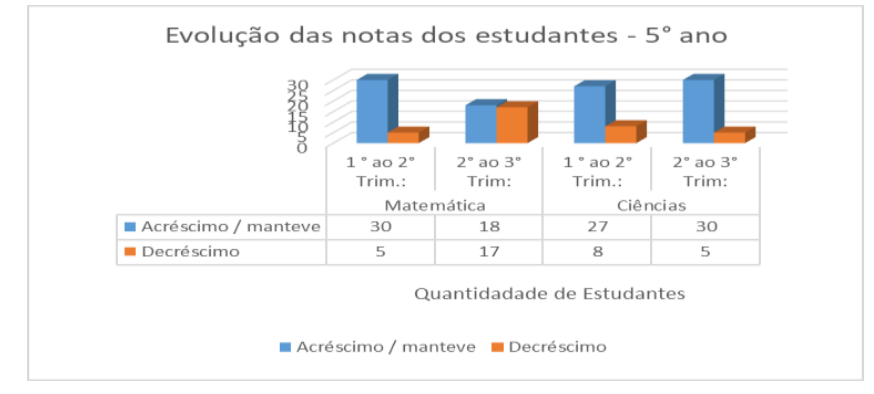

Gráfico 1. Evolução das Notas dos estudantes $-5^{\circ}$ ano

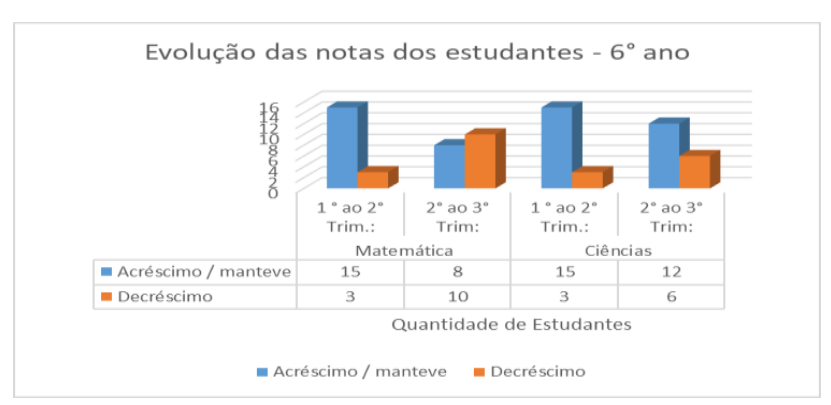

Gráfico 2. Evolução das Notas dos estudantes $-6^{\circ}$ ano

Para composição dos Gráficos 1 e 2 foram utilizadas tabelas com as notas das disciplinas de Matemática e Ciências das 2 séries atendidas que se referem as disciplinas das Ciências Exatas. As notas estão distribuídas entre $1^{\circ}, 2^{\circ}$, e $3^{\circ}$ Trimestres. A partir desses dados, foram realizadas comparações pontuais da seguinte maneira, nota do $2^{\circ}$ trimestre com Nota do $1^{\circ}$ trimestre, e nota do $3^{\circ}$ trimestre com nota do $2^{\circ}$ trimestre. Na análise foram considerados os seguintes critérios: Contagem das quantidades de notas aumentadas ou mantidas ficaram na coluna 'Acréscimo / manteve' e Quantidade de notas diminuídas ficaram na coluna 'Decréscimo'.

Os dados demonstraram que no caso do $5^{\circ}$ ano, as notas foram aumentando gradativamente conforme os trimestres foram sendo concluídos. Na disciplina de matemática foi observado melhora no rendimento escolar em $86 \%$ e $52 \%$ respectivamente, já na disciplina de Ciências foi observado melhora no rendimento escolar em $77 \%$ e $86 \%$ respectivamente.

Em relação aos dados do $6^{\circ}$ ano, com uma exceção na disciplina de Matemática com uma diferença percentual baixa, as notas também foram aumentando gradativamente entre os trimestres. Na disciplina de matemática foi observado melhora no rendimento escolar em $84 \%$ e $45 \%$ respectivamente, já na disciplina de Ciências foi observado melhora no rendimento escolar em $84 \%$ e $67 \%$ respectivamente.

\section{Considerações Finais}

Com o desenvolvimento das atividades, se percebeu, na prática, o quanto o uso dos jogos educativos tem se mostrado uma estratégia de ensino promissora.

O projeto apresentado está com suas atividades em andamento e ainda está tendo seus primeiros resultados coletados, avaliados e amplamente discutidos. Até o final de 2017, com o Ensino Fundamental foram realizados 23 encontros com cada turma. O retorno obtido dos monitores que apoiam as atividades, dos professores envolvidos, dos estudantes atendidos, dos pais e/ou responsáveis dos estudantes e da equipe pedagógica 
VII Congresso Brasileiro de Informática na Educação (CBIE 2018)

Anais do XXIX Simpósio Brasileiro de Informática na Educação (SBIE 2018)

da escola que vêm acompanhando de as atividades desenvolvidas, tem sido bastante positivo. $\mathrm{O}$ aumento gradativo das notas dos estudantes é notório. Esse é um resultado positivo significativo considerando que o projeto visa reforço pedagógico, especialmente nas disciplinas das Ciências Exatas.

Por fim, a iniciativa do projeto se mostrou interessante uma vez que os estudantes se identificaram e se envolvem emocionalmente, propiciando uma aprendizagem significativa, principalmente na disciplina de Matemática, que na maioria das vezes, os mesmos apresentam maior dificuldade no aprendizado. Assim, percebe-se que o uso das tecnologias computacionais e lúdicas na educação traz benefícios inquestionáveis.

\section{Referências Bibliográficas}

Andrade, A.; Carvalho, T.;Silveira, J.; Cavalheiro, S.; Foss, L.; Marilza, Fleischmann, A.; Aguiar, M.; and Reiser, R. (2013). "Proposta de Atividades para o Desenvolvimento do Pensamento Computacional no Ensino Fundamental". In XIX Workshop de Informática na Escola. P.169-178.

Barr, V. \& Stephenson, C. (2011). Bringing computational thinking to k-12: What is involved and what is the role of the computer science education community? ACM Inroads, 2(1):4854.

Campos, G.; Cavalheiro, S.; Foss, L.; Pernas, A.; Piana, C.; Aguiar, M.; Bois, A.; and Reiser, R. (2014). "Organização de Informações via Pensamento Computacional: Relato de Atividade Aplicada ao Ensino Fundamental". In XX Workshop de Informática na Escola. P.390-399.

FORBELlONE, A.L. V.; EBERSPACHER, H. F. Lógica de Programação. São Paulo, ed. Pearson Prentice Hal, 2005.

INEP. Relatório Censo da Educação Superior, 2016. Disponível em : http://download.inep.gov.br/educacao superior/censo superior/documentos/2016/notas sob re o censo da educacao superior 2016.pdf.

JÚNIOR, J. C. R. P.; RAPKIEWICZ, C. E.; DELGADO, C.X.; MOREIRA, J. A. Ensino de Algoritmos e Programação: Uma Experiência no Nível Médio. XXV Congresso da Sociedade Brasileira de Computação. Unisinos - São Leopoldo/RS. p. 2351-2362, 2005.

PINHO, G.; WEISSHAHN, Y.; CAVALHEIRO, S.; REISER, R.; PIANA, C.; FOSS, L.; AGUIAR, M.; and BOIS, A. D. 2016. "Pensamento Computacional no Ensino Fundamental: Relato de Atividade de Introdução a Algoritmos". In XXII Workshop de Informática na Escola. P.261-270.

; WEISSHAHN, Y.; CAVALHEIRO, S.; CAVALHEIRO, G.; and PIANA, C. F. de B. 2016. "Proposta de Jogo Digital para Dispositivos Móveis: Desenvolvendo Habilidades do Pensamento Computacional”. In XXVII Simpósio Brasileiro de Informática na Educação. P.100-109.

ROCHA, P. S.; FERREIRA, B., MONTEIRO, D.; NUNES, D.S. C.; GOÉS, H. C. N. Ensino e Aprendizagem de Programação: Análise da plicação de Proposta Metodológica Baseada no Sistema Personalizado de Ensino. Renote - Revista Novas Tecnologias na Educação, Porto Alegre - RS, Brasil. v. 8, n. 3, 2010.

SILVA, D. P. da; SIDNEI, S. S.; JESUS, A. M. de; and SILVA, C. E. P. 2016. "Aplicação de Robótica na Educação de Forma Gradual para o Estímulo do Pensamento Computacional”. In V Congresso Brasileiro de Informática na Educação. P.1188-1197.

WING, J. M. (2006). Computational thinking. Communications of the ACM, 49(3):33-35. 